\title{
Studies on the Iron Nanoparticles Catalyzed Reduction of Substituted Aromatic Ketones to Alcohols
}

\author{
L. Parimala and J. Santhanalakshmi \\ Department of Physical Chemistry, University of Madras Maraimalai Campus, Chennai 600 025, India \\ Correspondence should be addressed to J. Santhanalakshmi; jslakshmi@yahoo.co.in
}

Received 5 February 2014; Accepted 10 April 2014; Published 11 June 2014

Academic Editor: Vijaya Rangari

Copyright (C) 2014 L. Parimala and J. Santhanalakshmi. This is an open access article distributed under the Creative Commons Attribution License, which permits unrestricted use, distribution, and reproduction in any medium, provided the original work is properly cited.

\begin{abstract}
Iron nanoparticles are synthesized and size characterized using HRTEM, FESEM, and XRD. Polyethylene glycol(PEG), carboxymethyl cellulose (CMC), and poly N-vinyl pyrrolidone (PVP) are used as nanoparticle stabilizers. The sizes of Fe nps are found to be $9 \mathrm{~nm}, 14 \mathrm{~nm}$, and $17 \mathrm{~nm} \pm 1 \mathrm{~nm}$ corresponding to PEG, CMC, and PVP stabilizers, respectively. The three different iron nanoparticles (Fe nps) prepared are used as catalysts in the hydrogenation reaction of various substituted aromatic ketones to alcohols with $\mathrm{NaBH}_{4}$. The progress of the reaction was monitored using time variance UV spectra. Kinetic plots are made from the absorbance values and the pseudo first order rate coefficient values are determined. Catalytic efficiency of the Fe nps is obtained by comparing the pseudo first order rate coefficient values, times of reaction, and \% yield. Fe-PEG nps was found to act as better catalyst than Fe-CMC nps and Fe-PVP nps. Also, effects of substituents in the aromatic ring of ketones reveal that $+\mathrm{I}$ substituents are better catalysed than -I substituents.
\end{abstract}

\section{Introduction}

Reduction reactions of carbonyl compounds to primary and secondary alcohols possess one of the important classes of organic reactions that are well used in synthetic chemistry [1-5]. Such reactions find immense applications in chemical industries related to fine chemicals, pharmaceuticals, perfumes, and agrochemicals. Transition metal catalyzed reduction reactions are considered as popular substitutes of platinum metal based catalysts. Cost effectiveness, abundance, stability, recyclability, environmentally benign, and relatively nontoxic are some of the reasons for the important role of tranisition metals in catalysis. Decades of research involve traditional catalysts for ketone hydrogenation reactions involving precious metals and their coordination complexes [6-12]. Rhodium and ruthenium complexes using chiral phosphines and amines as ligands show excellent catalytic activity towards asymmetric hydrogenation of prochiral ketones and other carbonyl compounds. However, these catalysts have limited applications because of their high cost and difficulty in the separation of products from chiral catalyst. There have been several attempts to develop iron catalysts for these kinds of reactions, because these would be cheaper and nontoxic $[13,14]$. In this regard, Chirik's, Beller's, and Nishiyama's groups have recently reported useful iron catalysts for the hydrosilation of aldehydes and ketones $[7,15]$ and their transfer hydrogenation. Efforts to find catalysts that do not require noble metals are always required.

With the emergence of the possibility of production of stable transition metal nanoparticles with enhanced catalytic activity, selectivity, and recyclability, it is an additional advantage when such metal nanoparticles are used as efficient catalysts. Particularly for hydrogenation reactions recently, many reports on the use of iron nanoparticles as catalysts have evolved [16]. In reaction catalysis such as asymmetric transfer hydrogenation of ketones, alkene, alkyne hydrogenation, carbonyl reductions, and hydrogenation of several functional groups such as aldehydes, ketones, imines, and amides, iron nanoparticles as catalysts are reported [17-21]. Ketone hydrogenation reactions have also been reported to be catalysed by Fe nps [14]. The differences in the catalytic activity and in the efficiency of the catalysis of these reactions catalysed by $\mathrm{Fe}$ nps can be correlated to the differences in the sizes of the $\mathrm{Fe}$ nps used as catalysts. Regarding metal nanoparticles catalysis, 


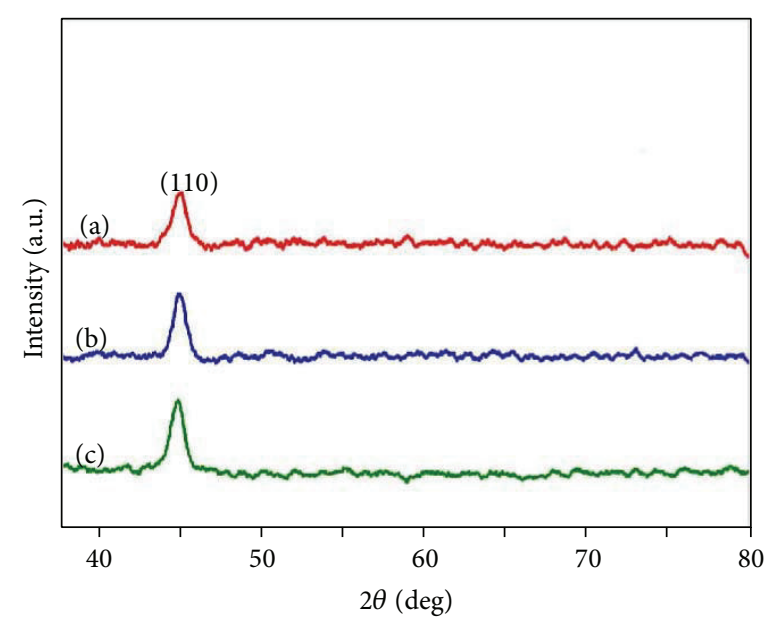

FIgURE 1: XRD patterns of Fe nps, having (a) PEG, (b) CMC, and (c) PVP as stabilizers.

aspects on particle size, ratio of surface area to volume per particle, surface exposure of atoms, and so forth prove useful to understand the nanocatalytic chemistry of the process. Zero valent $\mathrm{Fe}$ nps in the quantum dot range have been reported to be effective catalysts in many reduction reactions. Synthesis of Fe nps within 1-9 nm sizes are complex and tedious and involve sophisticated steps. In the present work, an inexpensive simple and one step preparation of Fe nps has been attempted and the use of biostabilizers for the Fe nps has been invoked. In many reports polyvinylpyrrolidone (PVP) has been chosen as the stabilizer. Stabilizers with biosource such as polyethylene glycol (PEG) and carboxymethyl cellulose (CMC) are chosen in the present work along with PVP. Aqueous hydrazine solutions as fresh stocks are used as the reducing agent in the iron salt precursor solutions. The sizes of the Fe nps each having PEG, CMC, and PVP as the stabilizer are characterized using XRD, FESEM, and HRTEM measurements.

The catalytic hydrogenation reactions of aromatic ketones with various ring substituents are performed using sodium borohydride as the reductant under ambient conditions. For each of the substituted ketone reactants, the progress of the reaction at regular intervals of time was studied using absorbance values at the UV characteristic wavelengths. Pseudo first order conditions are maintained and the kinetic parameters such as the overall pseudo first order rate coefficient values and overall product \% yield values are determined by repeating the reactions with the three different Fe np catalysts. The values are compared between the biostabilized and the synthetic polymer PVP stabilized Fe nps. Effects of $+\mathrm{I}$ and $-\mathrm{I}$ substituents in the aromatic ring of ketone substrates are studied based on the reaction parameters. In all cases, the products are separated and subjected to NMR analysis. The implications of the results obtained are discussed.

\section{Experimental}

2.1. Reagents. $\mathrm{FeCl}_{3}$ (anhydrous), $\mathrm{NaBH}_{4}$, aromatic ketones listed in Table 1 were analytical grade reagents and supplied by Sigma-Aldrich, India. The samples were used as such without further purification. The molecular weights of PEG, CMC, and PVP are 57-63, 90, and $40 \mathrm{KD}$, respectively, and are supplied by SRL, India. The reducing agent hydrazine hydrate, CTAB, and $\mathrm{NaOH}$ are purchased from Qualigens, India. Triple distilled water was used wherever necessary.

2.2. Synthesis of Iron Nanoparticles. Fe nps are synthesized using anhydrous $\mathrm{FeCl}_{3}$ as precursor, hydrazine hydrate as the reducing agent, and $\mathrm{PEG}, \mathrm{CMC}$, and $\mathrm{PVP}$ as the capping agents. Equal volumes of $0.01 \mathrm{M}$ metal salt, $0.01 \mathrm{M}$ reducing agent, and 3\% stabilizing agent solutions are mixed dropwise with constant stirring in a three-necked flask having a total volume of $250 \mathrm{~mL}$. After the addition, $10 \mathrm{~mL}$ of $0.01 \mathrm{M} \mathrm{NaOH}$ solution was added to maintain basic medium in the range of 10-11. Stirring was continued for 2 hours under nitrogen atmosphere. The Fe nps obtained as black precipitate was centrifuged and washed several times with ethanol and vacuum dried. The size of the particles was characterized using FESEM, HRTEM, and XRD measurements.

2.3. Particle Size Characterization. Powder X-ray analysis (XRD) was carried out using Bruker D8 Advance model diffractometer, operating at $40 \mathrm{kV}$ and $30 \mathrm{~mA} \mathrm{Cu} \mathrm{K \alpha}$ radiation with wavelength of $1.54 \AA$ and a step size of $0.02^{\circ}$ in the $2 \theta$ range; $10-80^{\circ}$ was used. Figure 1 presents the XRD profiles of Fe nps. FESEM and HR-TEM of the nanoparticles are measured using SU6600, HITACHI model operating at an accelerating voltage of $100 \mathrm{KV}$ and FEI-TECNAI $\mathrm{G}^{2}$, and model-T-30-S-twin operating at an accelerating voltage of $300 \mathrm{kV}$ instruments, respectively. The HR-TEM and FESEM photographs are shown in Figures 2 and 3. The morphology and the size of the nanocrystallites are measured from HRTEM images. The values coincided with those calculated from Scherrer formula using XRD data. $9.0 \mathrm{~nm}, 14.0 \mathrm{~nm}$, and $17.0 \mathrm{~nm} \pm 1.0 \mathrm{~nm}$ sizes are found for PEG, CMC, and PVP stabilized Fe nps, respectively.

2.4. Procedure for Hydrogenation of Aromatic Ketones. One pot batch reactor type procedure was adopted. Into a $200 \mathrm{~mL}$ 


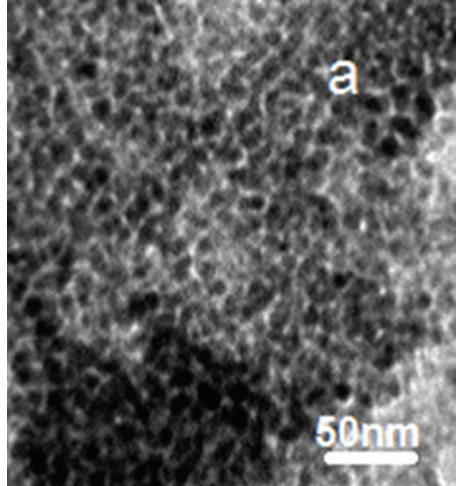

(a)

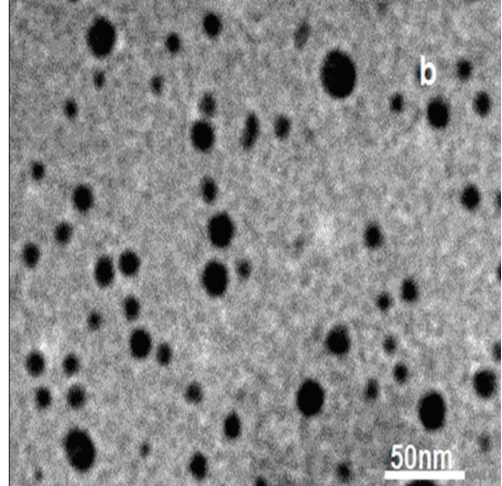

(b)

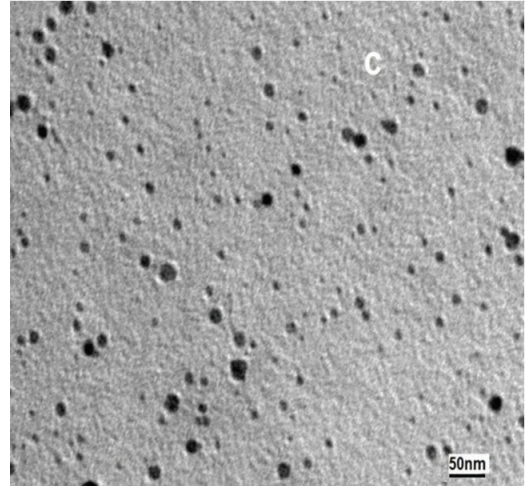

(c)

Figure 2: HRTEM image of (a) Fe-PEG nps, (b) Fe-CMC nps, and (c) Fe-PVP nps.

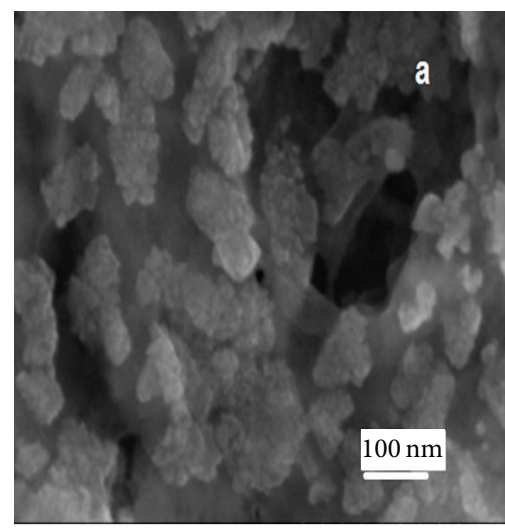

(a)

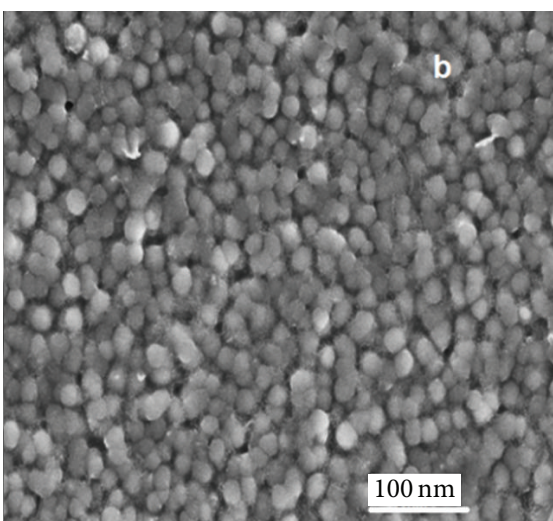

(b)

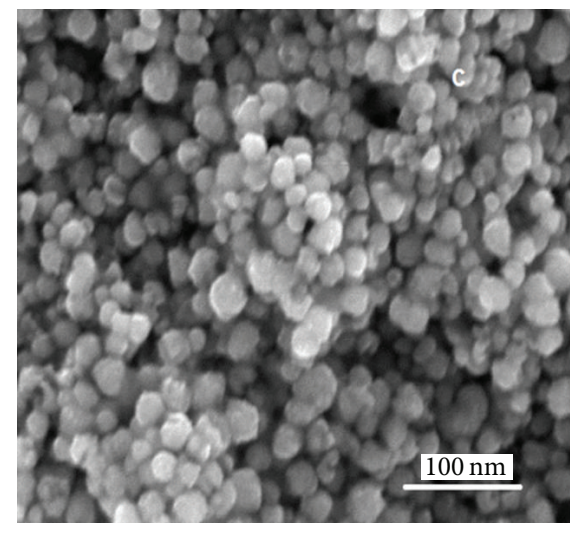

(c)

FIgUre 3: FESEM image of (a) Fe-PEG nps, (b) Fe-CMC nps, and (c) Fe-PVP nps.

capacity round bottomed four necked flask $1 \mathrm{mg}$ of the nanometal catalyst was added. Freshly prepared $1 \mathrm{mM} 5 \mathrm{~mL}$ $\mathrm{NaBH}_{4}$ solution, $0.1 \mathrm{mM}$ of $5 \mathrm{~mL}$ aromatic ketone solution, $0.1 \mathrm{mM} 2 \mathrm{~mL}$ CTAB solution, and $30 \mathrm{~mL}$ of triple distilled water were added dropwise from each neck of the flask with continuous stirring. The reaction mixture was maintained at $40^{\circ} \mathrm{C}$ for 1 hour. Although the reaction occurred at room temperature $\left(25^{\circ} \mathrm{C}\right)$, slight heating was maintained to ensure completeness of the reaction. At regular intervals of time, small aliquots are drawn out and UV spectra are recorded. The completion of the reaction was noted by the absorbance at the characteristic peak tracing the base line. The $\mathrm{H}^{1} \mathrm{NMR}$ spectra of the products are taken using $500 \mathrm{MHz}, \mathrm{BRUK}-$ ERAV III model instrument.

\section{Results and Discussion}

3.1. Morphology and Size Analysis of Fe nps. XRDs of Fe nps having PEG, CMC, and PVP as stabilizers are shown in Figure 1. The peak patterns are assigned to JCPDS data file number 01-1252 [22-24]. Applying the Scherrer formula to the peak, the sizes of the Fe nps with PEG, CMC, and PVP are found to be $8.5 \mathrm{~nm}, 13 \mathrm{~nm}$, and $16 \mathrm{~nm} \pm 1 \mathrm{~nm}$, respectively.
In Figure 2, HRTEM photographs of the Fe nps are given. The particles are spherical and nearly monodisperse. FESEM photographs are shown in Figure 3. From the HRTEM results, the average size of the particles was found to be $9 \mathrm{~nm}, 14 \mathrm{~nm}$, and $17 \mathrm{~nm}$, respectively. These values are in agreement with the XRD results. The trend in the sizes of Fe nps with various stabilizers in the increasing order was found to be Fe-PEGnp $<\mathrm{Fe}-\mathrm{CMC}<\mathrm{Fe}-\mathrm{PVP}$. When the interaction between the stabilizer and the metal nanoparticle is more, agglomeration of the particles will be less resulting in smaller sized particles. The formation of smaller sized Fe nps with PEG and CMC stabilizers compared to PVP stabilizer may be due to the strong interaction between the oxy(-O-) group of PEG and the hydroxy group of CMC with the Fe nps than the carbonyl group $(>\mathrm{C}=\mathrm{O})$ of PVP. This aspect shows that under similar experimental conditions $\mathrm{Fe}$ nps stabilized with biosource produced smaller sized particles with greater surface area than the synthetic polymer stabilizer PVP [25].

3.2. Catalytic Reduction of Aromatic Ketones. In the absence of Fe nps, reduction of aromatic ketones is quite slow. Thus, a combination of Fe np catalysts and $\mathrm{NaBH}_{4}$ is necessary to carry out the reduction at $30^{\circ} \mathrm{C}$. All reductions are carried out 
TABLE 1: Parameters of reduction reactions of aromatic ketones by $\mathrm{NaBH}_{4}$ with iron nanoparticles as catalysts $(\%$ yield $( \pm 0.5 \%)$ attained during reaction time $(\mathrm{h})$ ).

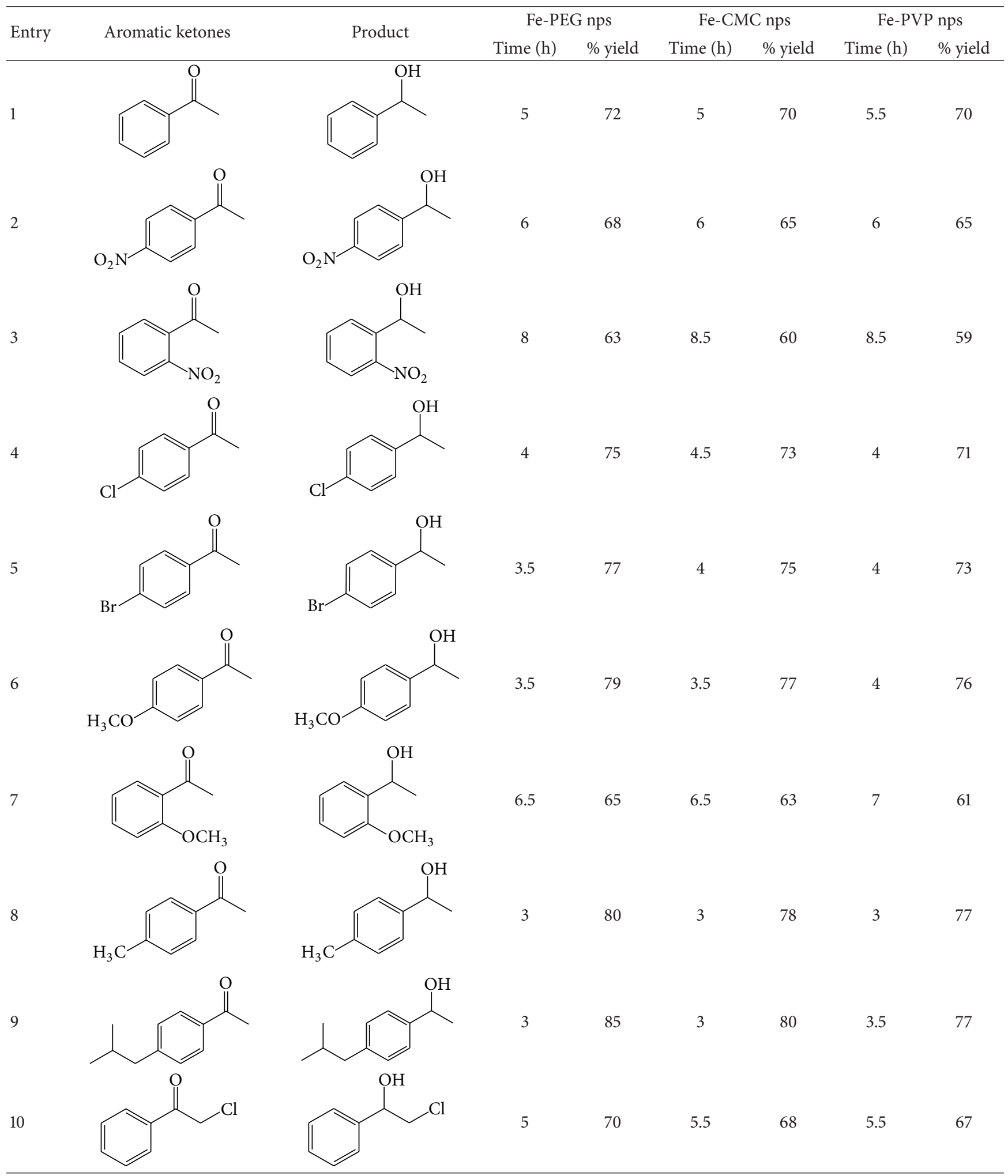


TABLE 2: Rate coefficient $k\left(\times 10^{-3} \mathrm{~min}^{-1}\right)$ values under pseudo first order conditions determined for the reduction reaction of aromatic ketones using $\mathrm{NaBH}_{4}$ and Fe nps with PEG, CMC, and PVP stabilizing agents.

\begin{tabular}{|c|c|c|c|c|}
\hline Entry & Aromatic ketones & $\begin{array}{c}\text { PEG } \\
k\end{array}$ & $\begin{array}{c}\mathrm{CMC} \\
k\end{array}$ & $\begin{array}{c}\text { PVP } \\
k\end{array}$ \\
\hline 1 & & 3.32 & 1.34 & 1.10 \\
\hline 2 & & 2.21 & 0.66 & 0.57 \\
\hline 3 & & 1.11 & 0.07 & 0.06 \\
\hline 4 & & 3.87 & 1.96 & 1.75 \\
\hline 5 & & 4.74 & 2.38 & 2.01 \\
\hline 6 & & 5.21 & 2.91 & 2.63 \\
\hline 7 & & 1.57 & 0.23 & 0.18 \\
\hline 8 & & 6.23 & 3.73 & 3.42 \\
\hline 9 & & 6.64 & 4.82 & 4.45 \\
\hline 10 & & 2.61 & 0.97 & 0.78 \\
\hline
\end{tabular}




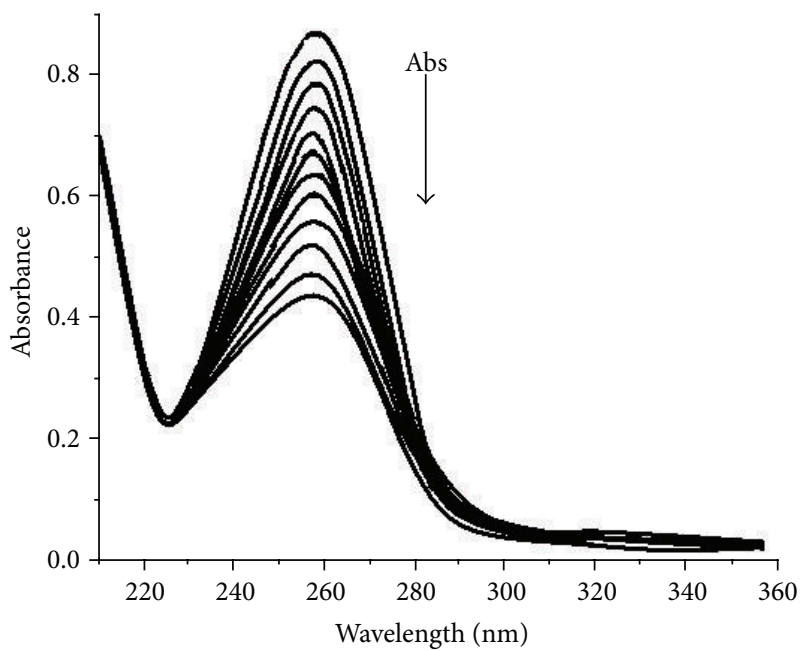

(a)

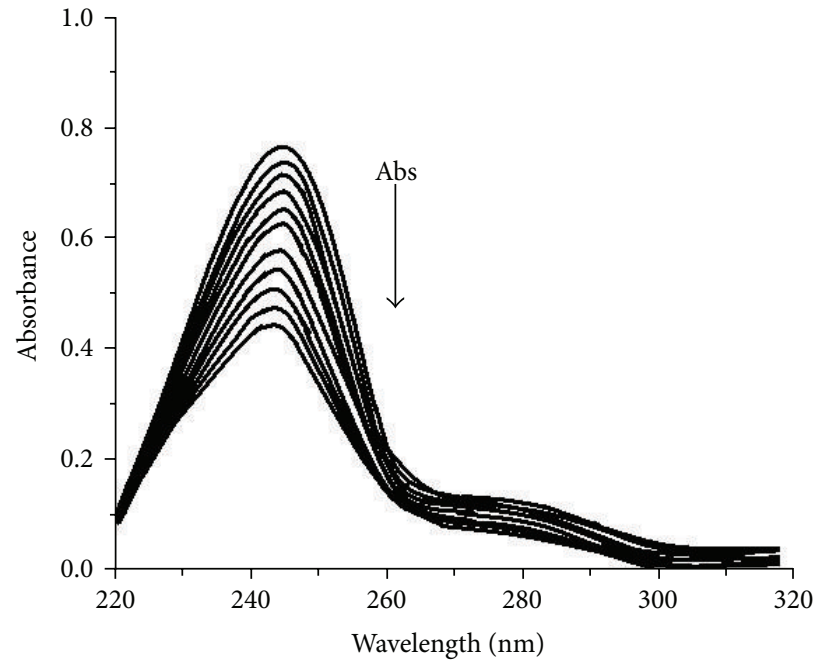

(c)

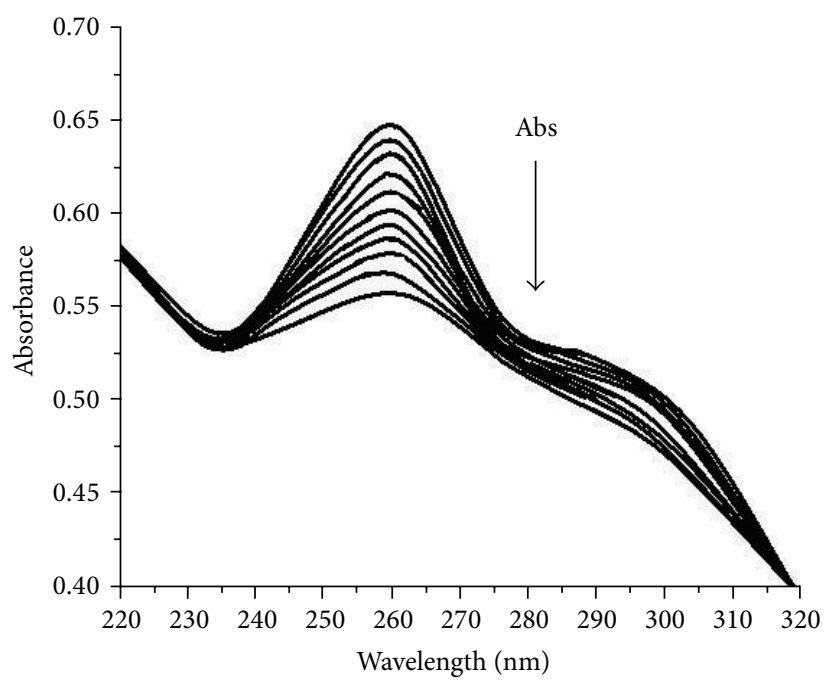

(e)

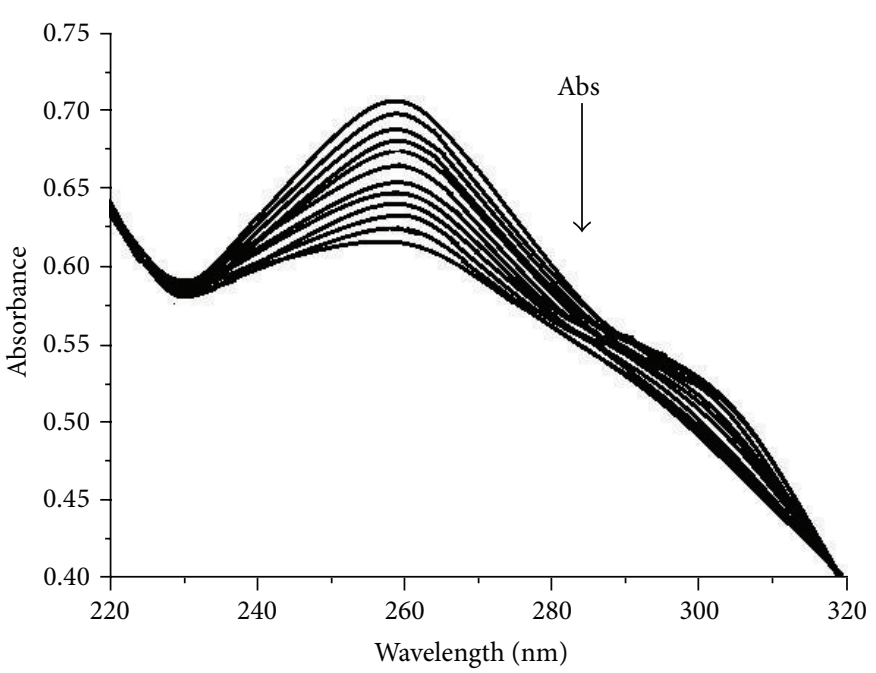

(b)

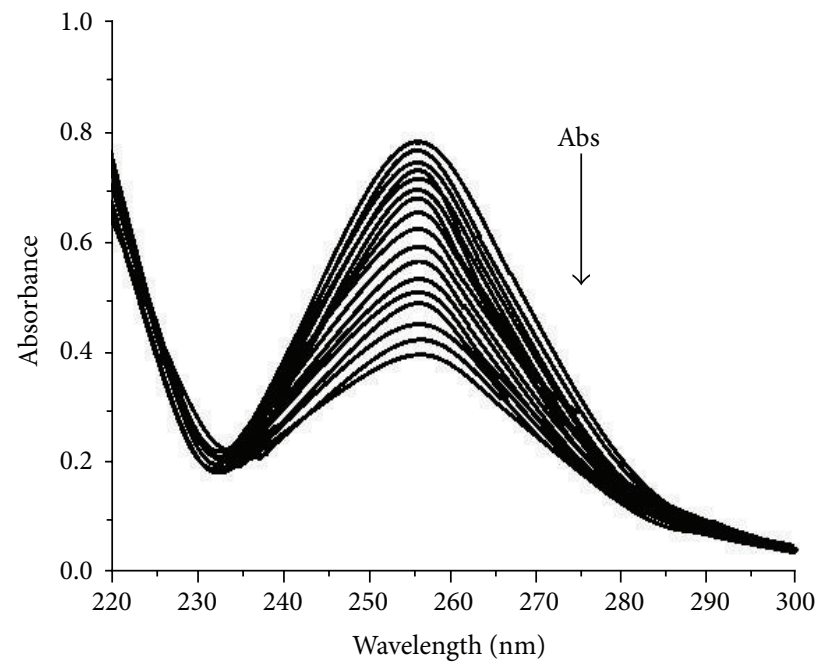

(d)

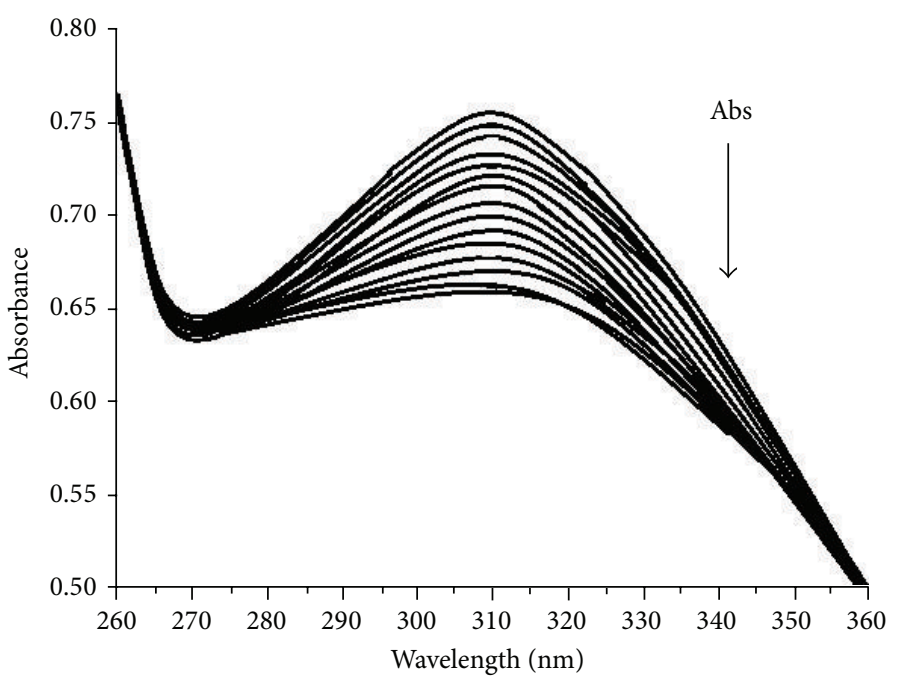

(f)

Figure 4: Time dependent UV-Vis spectra of reduction of aromatic ketones with $\mathrm{NaBH}_{4}$ (a) 1-(4-isobutylphenyl)ethanone, (b) 1-(4chlorophenyl)ethanone, (c) acetophenone, (d) 1-p-tolylethanone, (e) 1-(4-nitrophenyl)ethanone, and (f) 1-(4-methoxyphenyl)ethanone in the presence in the presence of Fe-PEG nps. 


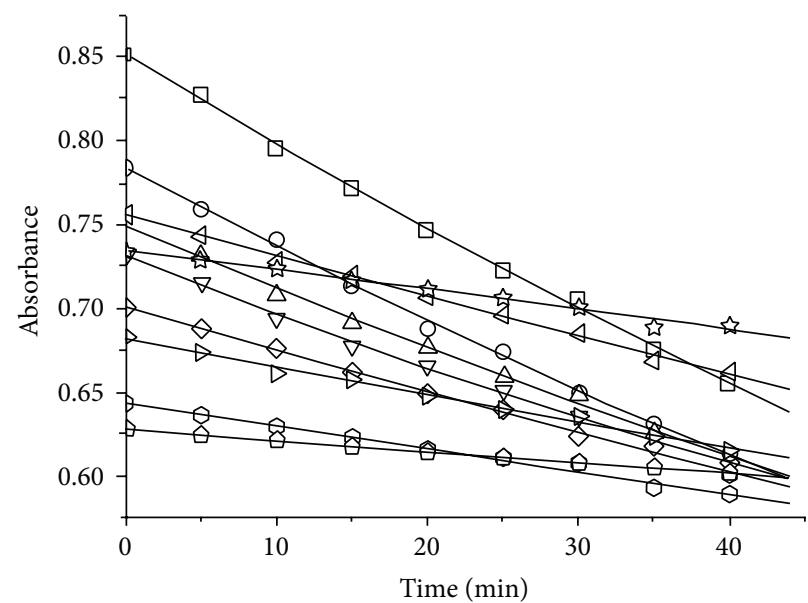

$\begin{array}{ll}\square(1) & \triangleleft(6) \\ \circ(2) & \triangleright(7) \\ \triangle(3) & \bigcirc(8) \\ \nabla(4) & \text { is }(9) \\ \diamond(5) & \square(10)\end{array}$
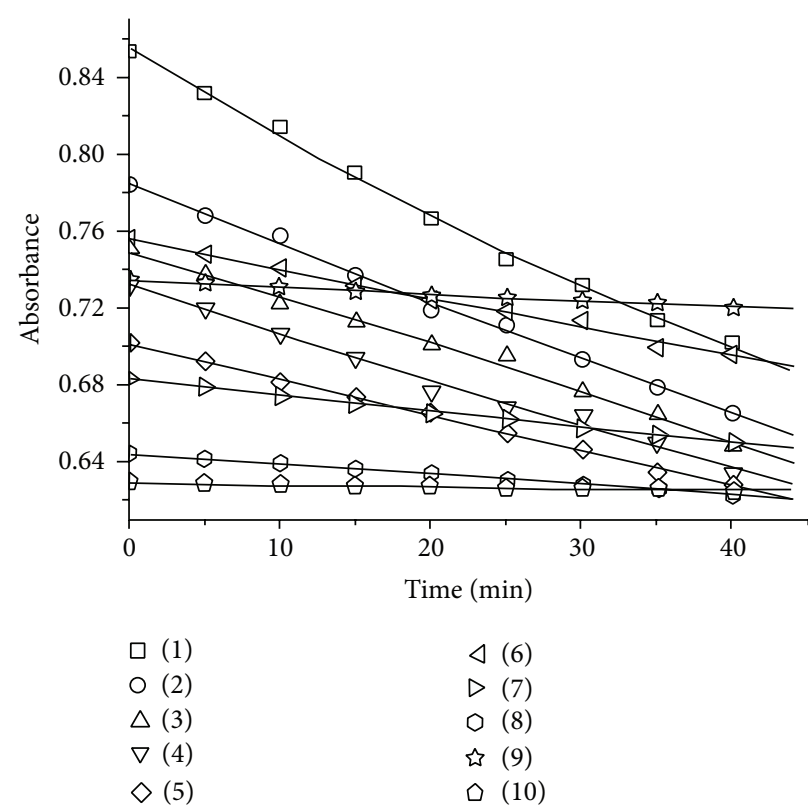

(b)

(a)

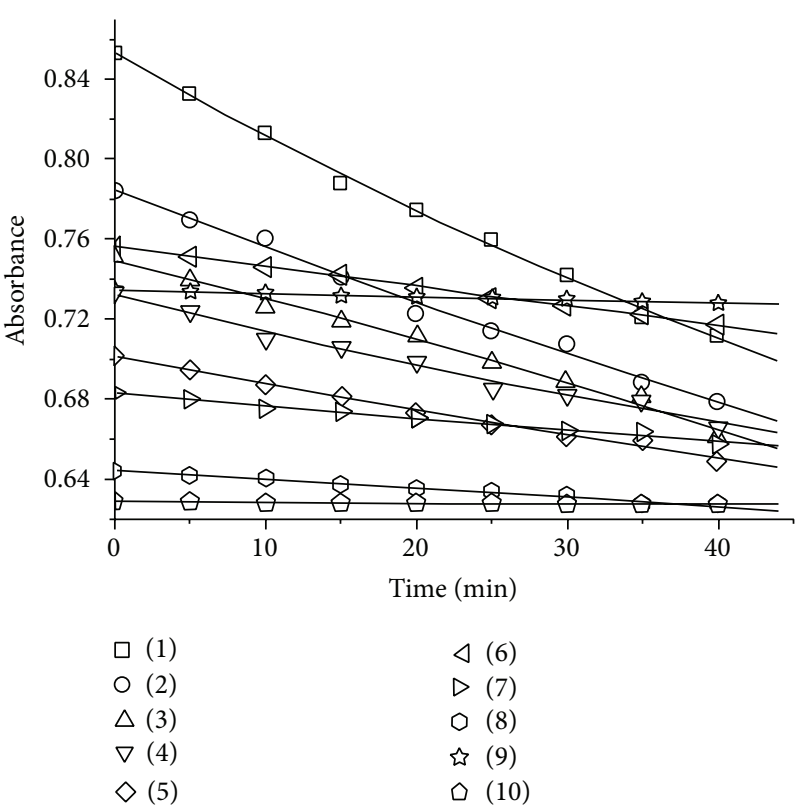

(c)

Figure 5: Absorbance time dependence plots for the reduction reactions of aromatic ketones with $\mathrm{NaBH}_{4}$ catalysed by (a) FePEG nanoparticles. (b) Fe-CMC nanoparticles. (c) Fe-PVP nanoparticles. (1) 1-(4-Isobutyl phenyl)ethanone, (2) 1-p-tolylethanone, (3) 1-(4-methoxyphenyl)ethanone, (4) 1-(4-bromophenyl)ethanone, (5) 1-(4-chlorophenyl)ethanone, (6) acetophenone, (7) 2-chloro-1phenylethanone, (8) 1-(4-nitrophenyl)ethanone, (9) 1-(2-methoxyphenyl)ethanone, and (10) 1-(2-nitrophenyl)ethanone.

with excess $\mathrm{NaBH}_{4}$ and no intermediate product was isolated in any reaction. The products isolated from solvent extraction technique are weighed and \% yield was calculated. Using TLC method, in all the reduction reactions the mentioned reduced product constitutes the major content. In Table 1, the nature of the product, \% yield, and times of reaction are listed for the three types of Fe nps employed as catalysts.

Fe nps stabilized with PEG, CMC, and PVP act as efficient catalysts in the reduction of aromatic ketones in the presence of $\mathrm{NaBH}_{4}$ aqueous solutions. In all the reductions listed in Table 1, Fe nps stabilized with PEG act as efficient catalyst than CMC and PVP stabilized Fe nps in terms of times of reaction to achieve nearly $80 \%$ yield. When the nanoparticle sizes are compared, it may be seen that Fe-PEG nps are smaller than Fe-CMC and Fe-PVP. Thus larger surface area to volume ratios is produced [26]. In addition, PEG and CMC polymers possess more oxy $(-\mathrm{O}-)$ and hydroxyl $(-\mathrm{OH})$ functional groups that efficiently bind the nascent hydrogen 


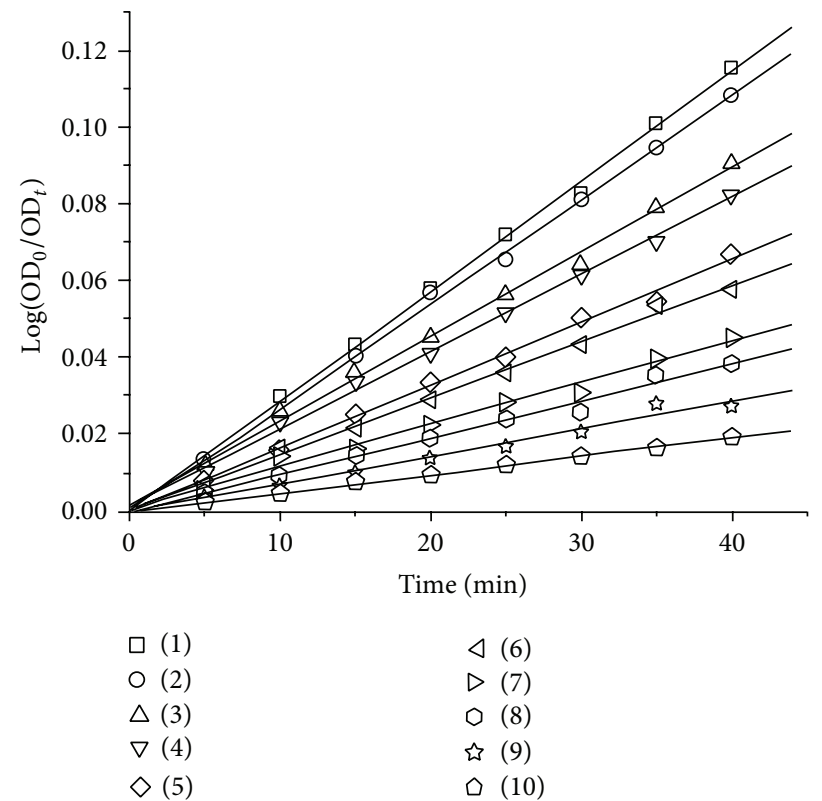

(a)

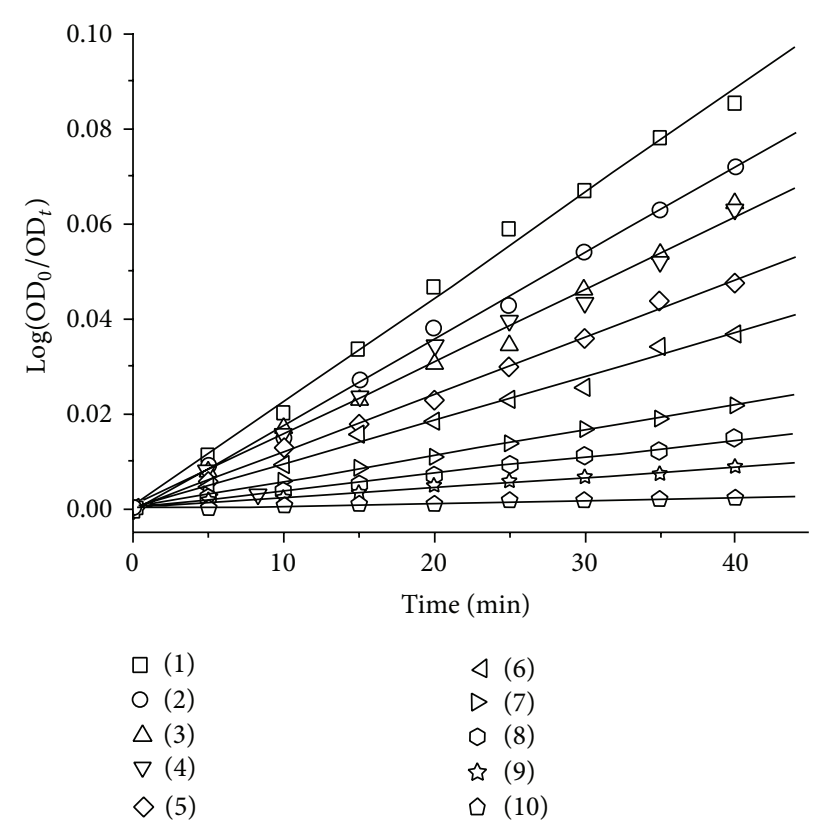

(b)

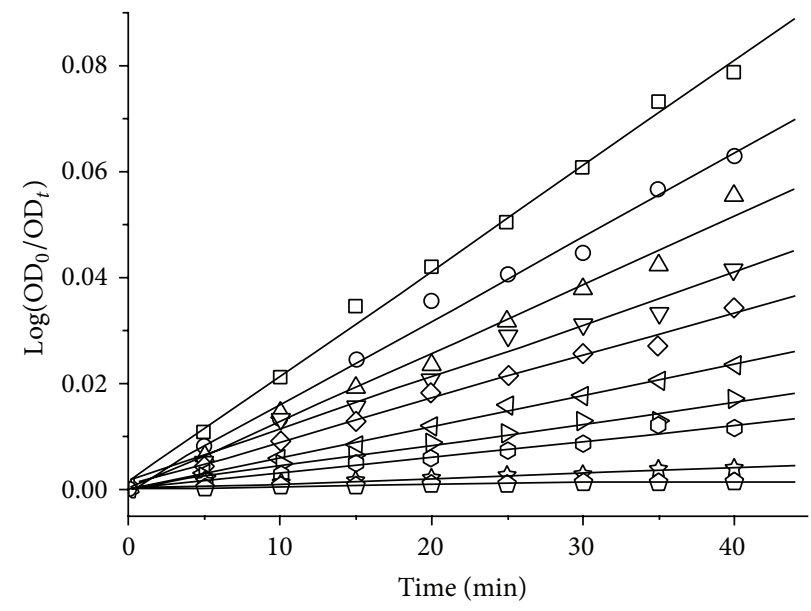

$\begin{array}{ll}\square(1) & \triangleleft(6) \\ \circ(2) & \triangleright(7) \\ \triangle(3) & \bigcirc(8) \\ \nabla(4) & \lesssim(9) \\ \diamond(5) & \square(10)\end{array}$

(c)

Figure 6: Kinetic plots for the rate coefficient determination of the reduction reactions of aromatic ketones with $\mathrm{NaBH}_{4}$ catalysed by (a) Fe-PEG nanoparticles. (b) Fe-CMC nanoparticles. (c) Fe-PVP nanoparticles. (1) 1-(4-Isobutyl phenyl) ethanone, (2) 1-p-tolylethanone, (3) 1-(4-methoxyphenyl)ethanone, (4) 1-(4-bromophenyl)ethanone, (5) 1-(4-chlorophenyl)ethanone, (6) acetophenone, (7) 2-chloro-1phenylethanone, (8) 1-(4-nitrophenyl)ethanone, (9) 1-(2-methoxyphenyl)ethanone, (10) 1-(2-nitrophenyl)ethanone.

produced by $\mathrm{NaBH}_{4}$ solution through H-bonding. PEG and $\mathrm{CMC}$ exhibit $\mathrm{H}$-bonding forces in addition to the capping and stabilizing effects of the Fe nps. In the case of PVP stabilized Fe nps, the carbonyl groups are engaged in stabilizing forces, while pyrrole ring nitrogen exhibits weaker $\mathrm{H}$ bonding interaction with the nascent hydrogen produced by $\mathrm{NaBH}_{4}$ solution than PEG and CMC nanoparticle stabilizers.
3.3. Kinetic Analysis. In Figure 4, time dependent UV spectra for six aromatic ketones chosen to represent the various substituents in Table 1 are given. Using time dependent UV spectra measurements, the absorbance versus time plot were made. The kinetic plots for the pseudo first order rate coefficient determination are produced by plotting $\log \mathrm{OD}_{0} / \mathrm{OD}_{t}$ versus time. Such plots are presented in Figures 5 and 6, 
respectively. The slopes of the linear plots are multiplied by 2.303 and produced the rate coefficient values. After the completion of the reaction the reaction mixture was filtered to remove the catalyst and the secondary alcohol products are extracted out using excess diethyl ether followed by evaporation with added anhydrous $\mathrm{Na}_{2} \mathrm{SO}_{4}$ to remove moisture. From the total amount of the product obtained, \% yield was calculated. In Tables 1 and 2, \% yield, the overall pseudo first order rate coefficient values, and times of reaction are given for various ring substituted aromatic ketones. The data indicate that the novel metal nanoparticle catalyst shows good catalytic performance for most of the aromatic ketones.

3.4. Effect of Aromatic Substituents. In Table 1, aromatic ketones corresponding to entries 3 and 7 contain osubstituents and show the effect of steric bulkiness on the catalysis performed in this work. Even for the o-substituents it may be seen that appreciable conversions and \% yields are found out. Also among the \% yield values reported psubstituents in the aromatic ring reactants have generated higher values than the o-substituted counter parts. Also when the substituents have $+\mathrm{I}$ effect (electron releasing nature) better and higher \% yield values are found compared to I substituents (electron withdrawing nature) in the aromatic ring.

The electronic effects and the metal nature in a combined way produce such experimental results. However the nature of the biostabilizer also influences the catalytic activity. From the $k$ values in Table 2, it may be seen that PEG biostabilized Fe nps show higher catalytic performance than the CMC stabilized ones. Higher rate coefficient values proportionate to the higher catalytic activity. Keeping all other conditions constant, the trend in the catalytic efficiency of the as synthesized Fe nps can be noted as PEG > CMC > PVP, nanoparticle system. In all cases only single products are detected. The alcohol products isolated are characterized by $\mathrm{H}^{1} \mathrm{NMR}$ spectra. The catalyst recovered after the completion of the reaction was dried well and recycled several times in many reaction repetitions. It was found that the rate coefficient values and the \% yield started to drop from fifth and sometimes sixth run onwards. When the catalyst recovered after the fifth run was examined under FESEM, intensely agglomerated particles are detected. This effect explains the loss of catalytic activity after fifth or the sixth run.

\section{Conclusions}

In conclusion, Fe nps are prepared with PEG, CMC, and PVP as stabilizers and are successfully used as catalysts for the hydrogenation of various substituted aromatic ketones, under mild and ecofriendlier experimental conditions. The resulting products from each run are easily separated from the catalyst system by extraction method. The catalyst recovered was reused in five or six times. Even-I effect substituents in the ketone produced appreciable yield values. The trend in the catalytic activity among metals falls in the line of decreasing size effect of the nanoparticles. That is, the order of the nanoparticle sizes increase as Fe-PEG $<\mathrm{Fe}-\mathrm{CMC}<\mathrm{Fe}-$ PVP. Fe-PEG produced smaller sized particles and produced higher performance than Fe-CMC and Fe-PVP nanoparticle systems. The biostabilised cost effective Fe nps have proved to be a better choice among the various catalysts available for the reduction of aromatic ketones.

\section{Conflict of Interests}

The authors declare that there is no conflict of interests regarding the publication of this paper.

\section{Acknowledgments}

The authors thank NCNSNT, University of Madras, for the FESEM and HRTEM results. L. Parimala thanks the University of Madras for the URF.

\section{References}

[1] J. W. Bae, S. H. Lee, Y. J. Jung, C. O. M. Yoon, and C. M. Yoon, "Reduction of ketones to alcohols using a decaborane/pyrrolidine/cerium(III) chloride system in methanol," Tetrahedron Letters, vol. 42, no. 11, pp. 2137-2139, 2001.

[2] Y. Ni and J. H. Xu, "Biocatalytic ketone reduction: a green and efficient access to enantiopure alcohols," Biotechnology Advances, vol. 30, no. 6, pp. 1279-1288, 2012.

[3] M. Aitali, S. Allaoud, A. Karim, C. Meliet, and A. Mortreux, "Enantioselective reduction of aromatic ketones catalysed by chiral ruthenium(II) complexes," Tetrahedron Asymmetry, vol. 11, no. 6, pp. 1367-1374, 2000.

[4] W. Kroutil, H. Mang, K. Edegger, and K. Faber, "Recent advances in the biocatalytic reduction of ketones and oxidation of sec-alcohols," Current Opinion in Chemical Biology, vol. 8, no. 2, pp. 120-126, 2004.

[5] A. Zanotti-Gerosa, W. Hems, M. Groarke, and F. Hancock, "Ruthenium-catalysed asymmetric reduction of ketones," Platinum Metals Review, vol. 49, no. 4, pp. 158-165, 2005.

[6] R. Langer, G. Leitus, Y. Ben-David, and D. Milstein, "Efficient hydrogenation of ketones catalyzed by an iron pincer complex," Angewandte Chemie, vol. 50, no. 9, pp. 2120-2124, 2011.

[7] N. S. Shaikh, S. Enthaler, K. Junge, and M. Beller, "Ironcatalyzed enantioselective hydrosilylation of ketones," Angewandte Chemie, vol. 47, no. 13, pp. 2497-2501, 2008.

[8] N. Meyer, A. J. Lough, and R. H. Morris, "Iron(II) complexes for the efficient catalytic asymmetric transfer hydrogenation of ketones," Chemistry, vol. 15, no. 22, pp. 5605-5610, 2009.

[9] R. Langer, M. A. Iron, L. Konstantinovski et al., "Iron borohydride pincer complexes for the efficient hydrogenation of ketones under mild, base-free conditions: synthesis and mechanistic insight," Chemistry, vol. 18, no. 23, pp. 7196-7209, 2012.

[10] T. Ohkuma, H. Ooka, H. Shohei, T. Ikariya, and R. Noyori, "Practical enantioselective eydrogenation of Aromatic Ketones," Journal of the American Chemical Society, vol. 117, pp. 2675-2676, 1995.

[11] C. A. Sandoval, T. Ohkuma, K. Muñiz, and R. Noyori, "Mechanism of asymmetric hydrogenation of ketones catalyzed by BINAP/1,2-diamine-ruthenium(II) complexes," Journal of the American Chemical Society, vol. 125, no. 44, pp. 13490-13503, 2003. 
[12] T. C. Johnson, W. G. Totty, and M. Wills, "Application of ruthenium complexes of triazole-containing tridentate ligands to asymmetric transfer hydrogenation of ketones," Organic Letters, vol. 14, no. 20, pp. 5230-5233, 2012.

[13] S. Gaillard and J. L. Renaud, "Iron-catalyzed hydrogenation, hydride transfer, and hydrosilylation: an alternative to preciousmetal complexes?" ChemSusChem, vol. 1, no. 6, pp. 505-509, 2008.

[14] J. F. Sonnenberg, N. Coombs, P. A. Dube, and R. H. Morris, "Iron nanoparticles catalyzing the asymmetric transfer hydrogenation of ketones," Journal of the American Chemical Society, vol. 134, no. 13, pp. 5893-5899, 2012.

[15] H. Nishiyama and A. Furuta, "An iron-catalysed hydrosilylation of ketones," Chemical Communications, no. 7, pp. 760-762, 2007.

[16] C. Rangheard, C. de Julián Fernández, P. H. Phua, J. Hoorn, L. Lefort, and J. G. De Vries, "At the frontier between heterogeneous and homogeneous catalysis: Hydrogenation of olefins and alkynes with soluble iron nanoparticles," Dalton Transactions, vol. 39, no. 36, pp. 8464-8471, 2010.

[17] P. H. Phua, L. Lefort, J. A. F. Boogers, M. Tristany, and J. G. De Vries, "Soluble iron nanoparticles as cheap and environmentally benign alkene and alkyne hydrogenation catalysts," Chemical Communications, no. 25, pp. 3747-3749, 2009.

[18] V. Kelsen, B. Wendt, S. Werkmeister, K. Junge, M. Beller, and B. Chaudret, "The use of ultrasmall iron(0) nanoparticles as catalysts for the selective hydrogenation of unsaturated C-C bonds," Chemical Communications, vol. 49, no. 33, pp. 34163418, 2013.

[19] A. Welther, M. Bauer, M. Mayer, and A. JacobivonWangelin, "Iron(0) particles: catalytic hydrogenations and spectroscopic studies," Chem CatChem, vol. 4, no. 8, pp. 1088-1093, 2012.

[20] R. Hudson, A. Rivière, C. M. Cirtiu, K. L. Luska, and A. Moores, "Iron-iron oxide core-shell nanoparticles are active and magnetically recyclable olefin and alkyne hydrogenation catalysts in protic and aqueous media," Chemical Communications, vol. 48, no. 27, pp. 3360-3362, 2012.

[21] M. Stein, J. Wieland, P. Steurer, F. Tölle, R. Mülhaupt, and B. Breit, "Iron nanoparticles supported on chemically-derived graphene: catalytic hydrogenation with magnetic catalyst separation," Advanced Synthesis and Catalysis, vol. 353, no. 4, pp. 523-527, 2011.

[22] K.-C. Huang and S. H. Ehrman, "Synthesis of iron nanoparticles via chemical reduction with palladium ion seeds," Langmuir, vol. 23, no. 3, pp. 1419-1426, 2007.

[23] L. Guo, Q. J. Huang, X. Y. Li, and S. Yang, "PVP-coated iron nanocrystals: anhydrous synthesis, characterization, and electrocatalysis for two species," Langmuir, vol. 22, no. 18, pp. 7867-7872, 2006.

[24] R. Singh, V. Misra, and R. P. Singh, "Synthesis, characterization and role of zero-valent iron nanoparticle in removal of hexavalent chromium from chromium-spiked soil," Journal of Nanoparticle Research, vol. 13, no. 9, pp. 4063-4073, 2011.

[25] J. Santhanalakshmi and L. Parimala, "The copper nanoparticles catalysed reduction of substituted nitrobenzenes: Effect of nanoparticle stabilizers," Journal of Nanoparticle Research, vol. 14, no. 9, article 1090, 2012.

[26] Y. Li, E. Boone, and M. A. El-Sayed, "Size effects of PVP$\mathrm{Pd}$ nanoparticles on the catalytic Suzuki reactions in aqueous solution," Langmuir, vol. 18, no. 12, pp. 4921-4925, 2002. 

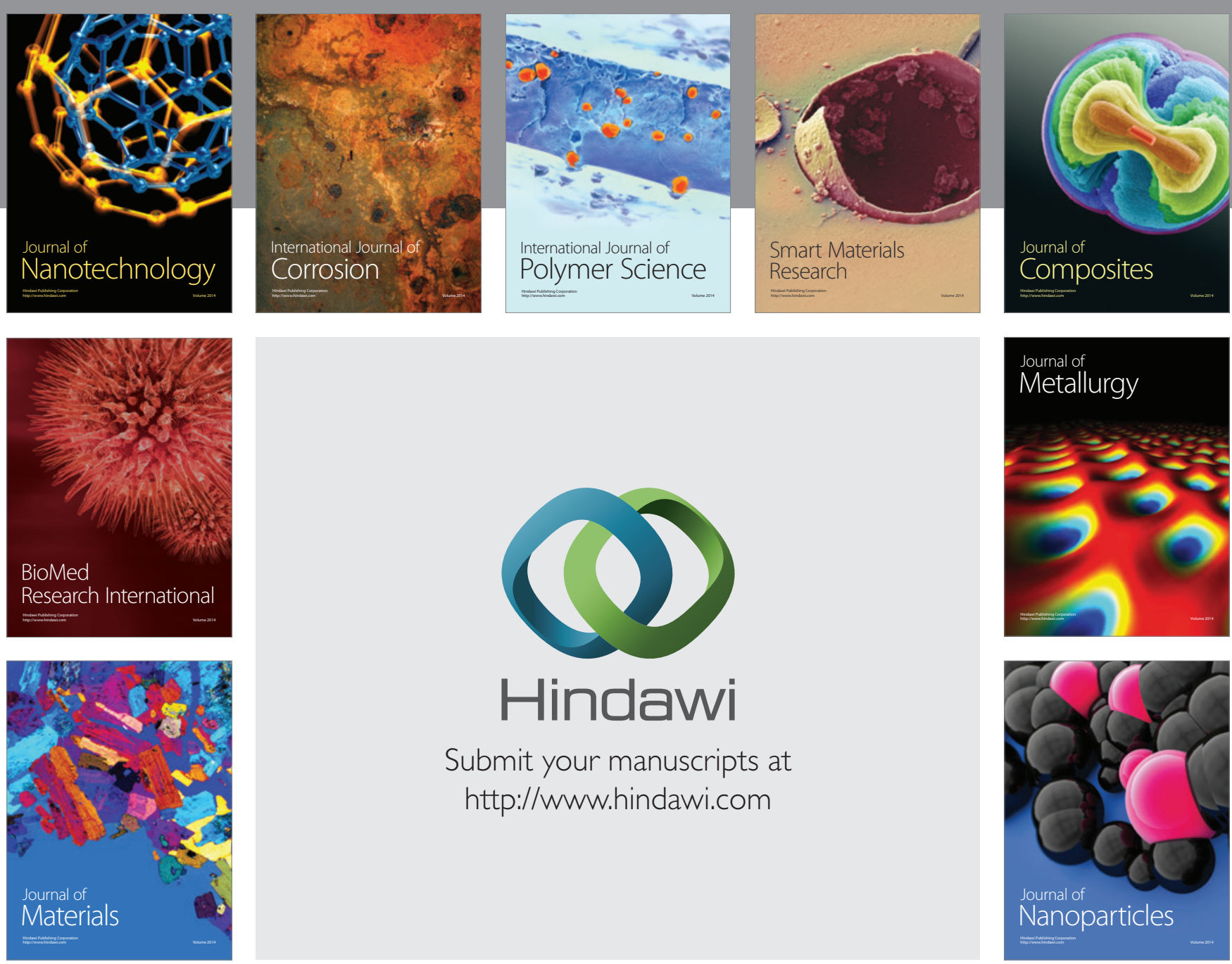

Submit your manuscripts at http://www.hindawi.com
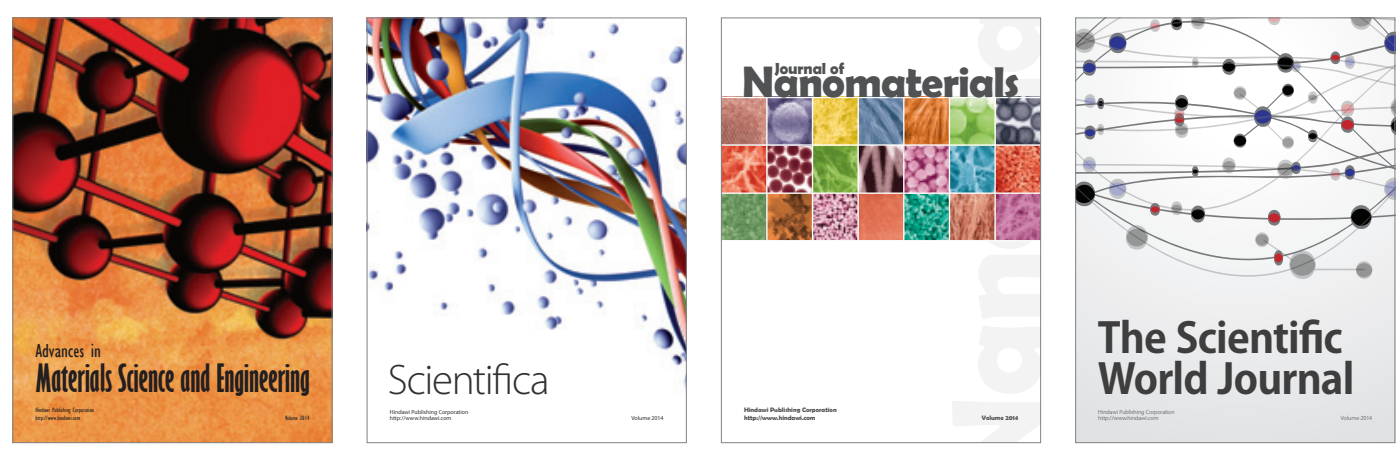

\section{The Scientific World Journal}
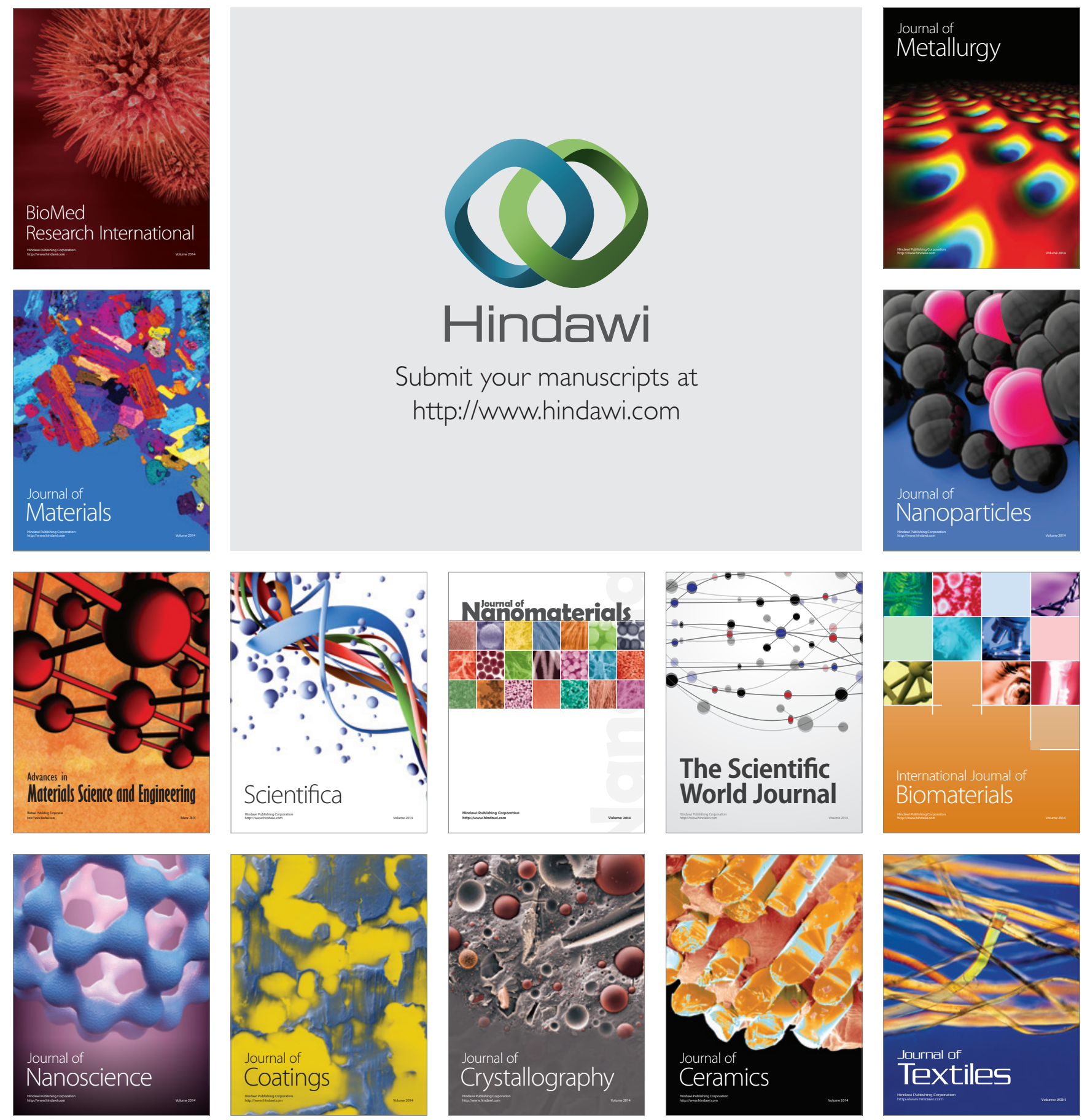